Technical Report TR-NCCA-2009-02

\title{
PROCEDURAL FUNCTION-BASED SPATIAL MICROSTRUCTURES
}

Alexander Pasko, Turlif Vilbrandt, Oleg Fryazinov, Valery Adzhiev

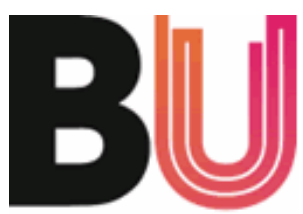

Bournemouth University

The National Centre for Computer Animation

Bournemouth Media School

Bournemouth University

Talbot Campus,

Poole, Dorset BH12 5BB

United Kingdom 


\begin{tabular}{|c|}
\hline Technical Report TR-NCCA-2009-02 \\
\hline ISBN: $1-85899-123-4$ \\
\hline Title: Procedural Function-based Spatial Microstructures \\
\hline Authors: Alexander Pasko, Turlif Vilbrandt, Oleg Fryazinov, Valery Adzhiev \\
\hline Key words and Phrases: Microstructure, Lattice, Porous Media, Tissue engineering, Scaffold, FRep, Digital fabrication \\
\hline Abstract: \\
\hline $\begin{array}{l}\text { We propose a new approach to modelling heterogeneous objects containing internal spatial geometric structures with } \\
\text { size of details orders of magnitude smaller than the overall size of the object. The proposed function-based procedural } \\
\text { representation provides a compact, precise, and arbitrarily parameterized model allowing for modelling coherent } \\
\text { microstructures, which can undergo blending, offsetting, deformations, and other geometric operations, and can be } \\
\text { directly rendered and fabricated without generating any auxiliary representations. In particular, modelling of regular } \\
\text { lattices and porous media is discussed and illustrated. Examples of microstructure models rendering and fabrication } \\
\text { using a variety of digital fabrication machines and materials are presented. }\end{array}$ \\
\hline Report date: April 27, 2009 \\
\hline Web site to download from: http://eprints.bournemouth.ac.uk/ \\
\hline $\begin{array}{l}\text { The authors’ e-mail addresses: apasko@bournemouth.ac.uk, turlif@turlif.org, ofryazinov@bournemouth.ac.uk, } \\
\text { vadzhiev@bournemouth.ac.uk }\end{array}$ \\
\hline Supplementary Notes: \\
\hline
\end{tabular}

\author{
The National Centre for Computer Animation \\ Bournemouth Media School \\ Bournemouth University \\ Talbot Campus, \\ Poole, Dorset BH 12 5BB \\ United Kingdom
}



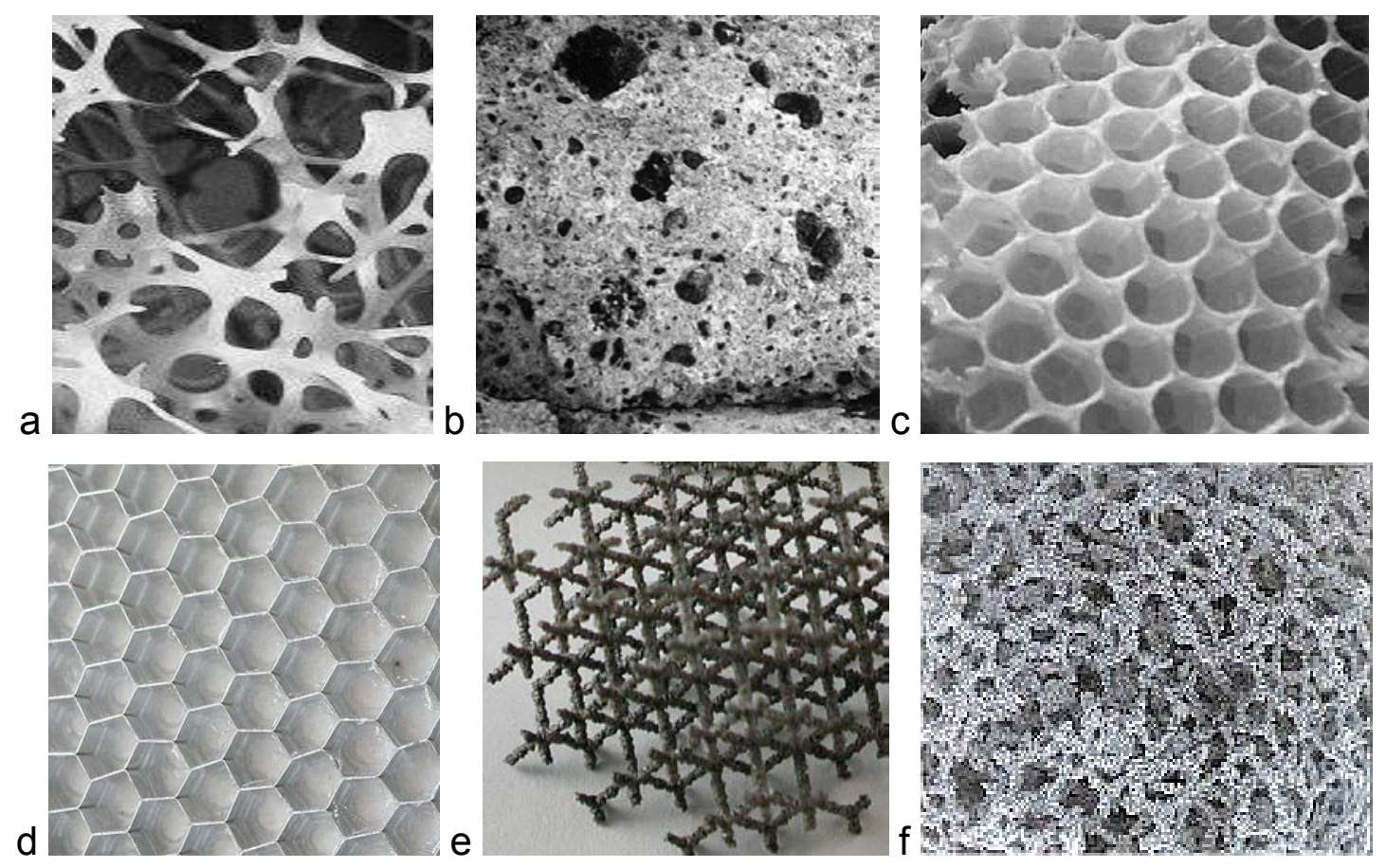

Figure 1. Natural and artificial microstructures: (a) bone; (b) bauxite ore; (c) bees honeycomb; (d) aluminium honeycomb; (e) metal lattice; (f) titanium scaffold.

\section{Introduction}

Recent developments in computing allow researchers to look beyond the traditional geometric modelling techniques oriented towards surface based and homogeneous solid models. Man-made objects are often nearly uniform in their internal structure, for example, most of mechanical parts can be modelled as homogeneous solids. In contrast, natural objects are rarely homogeneous having a complicated internal structure and materials distribution.

New application areas of CAD/CAM such as biomedical tissue engineering [SSN $\left.{ }^{*} 05\right]$ and composite materials in mechanical engineering [KT08] deal with essentially heterogeneous objects. Such objects can be made of different materials with variable densities and can have internal microstructures (see Fig. 1). The common feature of such objects is presence of internal spatial geometric structure with size of details orders of magnitude smaller than the overall size of the object. These can be lattices, porous, branching or granular material compositions varying from regular to completely irregular random structures. Not only are microstructures important in engineering new complex objects but are becoming increasingly important for manufacturing traditional objects using digital fabrication processes. Adding internal microstructures to objects reduces the weight, materials, energy and time required to create an object. In the future it is likely that most large, digitally fabricated objects will not be created as solid blocks of materials but will instead be made up of microstructures.

The existing approaches to modelling geometry of microstructures include those based on surfaces (boundary representations, BRep) and voxels (discrete volume representations). Although the researchers can to some extent tackle this problem using both these 
approaches (see the next section), generally many known problems and limitations of both representations are largely amplified by the factor of the geometric complexity of microstructures. One can distinguish between quantitative problems (model size and processing time) and qualitative problems (model validity, precision, parameterization, operability, and manufacturability) of existing representations:

\section{- Size and processing time}

Surface based models of a moderate size containing high quality lattice microstructures can include such a huge number of polygons that it becomes difficult or impossible for modern graphics hardware to render. Although the size of voxel models is not directly dependent on the object complexity, the number of voxels needed to represent high quality surfaces and small details can easily exceed available capacities of computer memory. Time for rendering and other types of processing grows with the size of the model.

\section{- Validity and precision}

Known problems arising from traditional BRep based CAD models and approaches - such as cracks in surfaces, self-intersections of polygons, additional false polygons left over from modelling, and inverted normal orientation - already problematic for the manufacturing industry, become increasingly difficult or impossible to model and manufacture at nano- and micro- scales. BRep and voxel models are usually created inside some specifically given bounding unit and then iteratively replicated in space without considering spatial coherence of the structure, which can cause additional cracks in a model at the boundaries of each unit. Likewise, the "skinning" or proper connection of a microstructure to the object shell presents many unsolved issues for current systems. Both the polygonal BRep and the voxel representation are not exact in the general case and only approximate the modelled geometry with limited precision.

\section{- Parameterization and operability}

The support of model generation with variable parameters is crucial for modelling microstructures, because their geometry can depend on a number of factors such as distance to the object surface, predefined strength conditions, density variations, and others. The existing representations have limited or no support for parameterization of microstructure models. When parameters are changed, BRep and voxel models have to be re-generated using some higher level generation procedure. A user may need to apply further specific operations on microstructures such as offsets, blends and shape deformations, which have limited or no support within the current representations.

- Manufacturability

Controlled complex microstructures are not easy to manufacture using most existing technologies. Currently the best solution can be found in rapid prototyping or digital fabrication technologies such as laser sintering, stereolithography and other additive processes, which produce a physical object layer by layer [MH03, CYL05, NCL ${ }^{*} 05$, $\left.\mathrm{SSN}^{*} 05\right]$. As object becomes large or if very fine microstructures are required, BRep models dramatically increase in size and become very hard or impossible for current hardware systems to visualize or cross-section as is required by many digital fabrication systems. While current digital fabrication systems have limited resolutions, in recent years they have achieved ever greater accuracy and this trend is expected to continue. Even so, it is not uncommon to see defects or missing sections in objects produced largely due to the complexity in creating proper cross-sections from the STL format. Some but not all digital fabrication systems accept voxels models as input however voxles have known aliasing 
problems unless they at very high resolutions requiring large amounts of memory. For systems that do not work with voxels, isosurfaces of interest have to be extracted and polygonized.

The problem addressed in this work is to develop an approach to modelling microstructures resolving most of the above problems. We propose a compact, precise, and arbitrarily parameterized model allowing for the procedural generation of spatially coherent microstructures, which can undergo blending, offsetting, deformations, and other geometric operations, and can be directly manufactured without generating any auxiliary representations.

We concentrate in this work on modelling lattice and porous media microstructures. Under "lattice" we mean a periodical spatial structure consisting of crossing rods, laths or other thin strips of material (Fig. $1 \mathrm{c}, \mathrm{d}, \mathrm{e}$ ). The term "porous media" describes various materials that exhibit the property of porosity through an interconnected network of cavities (pores) within a solid material stratum (Fig. 1 a,b,f). We try to avoid using the term "scaffold", because it can be applied to both types of structures.

\section{Other works}

In this section we first describe the main approaches to modelling microstructures and then introduce the basics of the function representation for geometric models.

\subsection{Lattices}

Generation of a polygonal model of a regular lattice with cubic elements for visualization is quite trivial using standard surface primitives such as cylinders or blocks. The problem becomes more complex if a valid solid BRep model is required for further operations on it such as intersection with the given overall shape of the object, and blending between the lattice rods and the outer surface. Existing publications provide quite limited information on procedures of the lattice models generation and mainly concentrate on providing application specific properties of lattices.

A geometric modelling method for creating conformal lattice structures was proposed in [WCR05]. Lattice structures are a built as a set of unit trusses. The solid model of each unit truss is created and Boolean operations are performed in a commercial solid modelling system. Meshes of unit trusses are then stacked together directly to generate the model of the entire lattice structure. However, it was pointed out that it took significant computational resources to generate the models of lattice structures since the generation of each unit truss required several Boolean operations. The maximal practically achievable number of lattice struts was 2400. This work was extended in [C07] to the general internal structure design. The proposed 3D texture mapping approach is based on mapping of a 3D microstructure pattern into a design space to generate internal structures which then were combined with a given CAD model of the object.

A classification of lattices is given in [SSN*05] along with the outline of the modelling process in biomedical applications involving predefined lattice patterns and scanned biological shapes such as human bones. 
The work $\left[\mathrm{NCL}^{*} 05\right]$ describes typical steps of lattice design in tissue engineering and fabrication. A commercial BRep solid modelling system is used to generate lattices for bone implants with selected spatial structure parameterized by pore sizes, porosity, and surface area to volume ratio. The overall required shape of the model is taken from human body data collected by magnetic resonance imaging. The Boolean intersection is applied to produce a lattice within the patient's bone shape. A surface triangulation in the form of the STL file is performed for the fabrication step. The designed microstructures were fabricated using a powder-based rapid prototyping technique called selective laser sintering.

Optimization of lattices is presented in [MH03, GSR05, H07] using maximal strength, minimal weight, and other criteria. The lattice structure (namely, connections between lattice nodes and positions of nodes) is optimized to satisfy the selected criteria.

\subsection{Porous media}

Modelling porous media is much more complex than modelling lattices because of irregularities essential to pores' locations and shapes. It can be stated that available CAD methods and tools are not adequate for solving this problem. There are two main approaches pursued in recent works: image-based reconstruction [SSN*05, VAG*08] and pseudo-random simulation [LS04, SRS*05, CTS07].

The process of obtaining geometric surface models for biological porous tissues is described in [SSN$\left.{ }^{*} 05\right]$. It includes obtaining $2 \mathrm{D}$ images of the internal tissue structure using computer tomography and other techniques; reconstruction of a 3D voxel model from a set of images; and construction of a surface model through the tessellation of a selected isosurface or by the voxel model segmentation and fitting with predefined parameterized surface primitives.

To reconstruct a 3D model of a porous structure, the authors of [VAG*08] construct a voxel model from a set of cross-section images of a biological material sample. The voxel model is segmented into three regions: exterior, material and pores. A graph is built reflecting individual pores and connections between them. A geometric model can be generated and visualized where each pore is represented by a sphere with the estimated radius.

A direct approach to 3D modelling of porous media is presented in [LS04]. First, a number of microspheres, which can be packed within the given volume, is estimated. Then the selected number of microspheres is randomly packed in the volume. The Boolean subtraction of the microspheres model from the basic volume model using a commercial BRep modeller results in the porous volume structure. The experiments were conducted with a quite limited number of microspheres (about 50 ).

A model of a porous object is represented in [SRS*05] as a Constructive Solid Geometry (CSG) tree with stochastically generated primitives (spheres) representing pores in the leaves of the tree structure and regularized Boolean operations placed in its nodes. The structure analysis is performed on the voxelized version of this model.

A 3D porous structure can be built as a stack of thin extruded $2 \mathrm{D}$ slices. The structure in each slice is modelled in [CTS07] using a Voronoi diagram built for the structure generating points moving on the plane. 
We can conclude that BRep and voxels remain the main models researchers use to represent microstructures. The major drawbacks of these representations were discussed in the previous section. In the remainder of the paper we tackle these problems using procedural function-based models in the form of the function representation (FRep).

\subsection{FRep basics}

The main idea of the function-based modelling (or "implicit surface" modelling [ $\left.\mathrm{B}^{*} 97\right]$ ) is to define point sets via trivariate scalar fields or continuous real functions of point coordinates $F(x, y, z)$. The point membership depends on the sign of the defining function evaluated at that point, typically points with positive and zero function values are included in the set, while zero-level subset is considered a boundary. The distinguishing key feature of this model is that the defining function cannot and has not to be preliminary evaluated at any point, the model implementation is a procedure providing the function evaluation at the given point upon request during the model interrogation in rendering, analysis, or conversion to other representations. This approach to geometric modelling was extended in the Function Representation (FRep) [PAS*95] by explicitly introducing elementary objects (primitives) and operations on them with corresponding operations on defining functions. This provides a basis for a modelling system for the creation and manipulation highly complex and heterogeneous function-based objects. Our experimental models in this paper are implemented in the HyperFun language [ACF*99], which fully supports the FRep modelling paradigm. Among the FRep advantages one can notice the possibility of direct rendering [FP08] and fabrication [VML*08] of created models without their conversion to BRep, voxel or other auxiliary representations (see details in later sections).

One particular class of the FRep operations are set-theoretic ones defined by R-functions [R74, S88, PAS*95, S07]. An object resulting from the set-theoretic operations has the defining function expressed as follows:

$f_{3}=f_{1} \vee_{\alpha} f_{2}$ for the union;

$f_{3}=f_{1} \wedge_{\alpha} f_{2}$ for the intersection,

$f_{3}=f_{1} \backslash_{\alpha} f_{2}$ for the subtraction,

where $f_{1}$ and $f_{2}$ are defining functions of initial objects and $\vee_{\alpha}, \wedge_{\alpha}$ are signs of R-functions.

One of the classes of R-functions is

$f_{1} \vee_{1} f_{2}=\max \left(f_{1}, f_{2}\right)$
$f_{1} \wedge_{1} f_{2}=\min \left(f_{1}, f_{2}\right)$
$f_{1} \backslash_{1} f_{2}=\min \left(f_{1},-f_{2}\right)$

These functions are $C^{1}$ discontinuous at all points where $f_{1}=f_{2}$. R-functions of another class:

$$
\begin{aligned}
& f_{1} \vee_{0} f_{2}=f_{1}+f_{2}+\sqrt{f_{1}^{2}+f_{2}^{2}} \\
& f_{1} \wedge_{0} f_{2}=f_{1}+f_{2}-\sqrt{f_{1}^{2}+f_{2}^{2}} \\
& f_{1} \backslash_{0} f_{2}=f_{1}-f_{2}-\sqrt{f_{1}^{2}+f_{2}^{2}}
\end{aligned}
$$

have $C^{1}$ discontinuity only at the points where both arguments are equal to zero.

Shapiro [S88] showed that a periodical regular structure such as a chess board can be modeled using trigonometric functions defining horizontal and vertical strips, which then are 
combined using the set-theoretic union. We further develop this idea to model 3D microstructures and to apply operations to them.

\section{Modelling lattice microstructures}

We propose the following approach to modelling infinite regular 3D lattices:

1) a set of infinite parallel slabs orthogonal to each coordinate axis can be defined by a corresponding periodic function;

2) the intersection of two of these sets results in the infinite rods parallel to one of the axes;

3) the union of rods gives us an infinite rectangular lattice.

To define the infinite slabs, the following functions can be applied:

$s_{x}(x, y, z)=\sin \left(q_{x} x+p_{x}\right)-l_{x}$

$s_{y}(x, y, z)=\sin \left(q_{y} y+p_{y}\right)-l_{y}$

$s_{z}(x, y, z)=\sin \left(q_{z} z+p_{z}\right)-l_{z}$

where the inequality $s_{x} \geq 0$ describes a set of slabs orthogonal to $x$-axis and parallel to each other, the frequency $q_{x}$ defines the distance between parallel slabs along $x$-axis, the phase $p_{x}$ defines the position of slabs on the $x$-axis relative to the origin, and the threshold $-1<I_{x}<$ 1 together with the frequency defines the thickness of each slab. The slabs orthogonal to $y$ and $z$ axes are symmetrically defined by the functions $s_{y}$ and $s_{z}$.

The next step is to describe three sets of rods parallel to each axis:

$r_{x}(x, y, z)=s_{y} \wedge_{\alpha} s_{z}$

$r_{y}(x, y, z)=s_{x} \wedge_{\alpha} s_{z}$

$r_{z}(x, y, z)=s_{x} \wedge_{\alpha} s_{y}$

Here the inequality $r_{x} \geq 0$ describes a set of rods parallel to $x$-axis and obtained as the settheoretic intersection between slabs orthogonal to $y$-axis and $z$-axis using an R-function (Eqs. 1-3). The final infinite regular lattice as a union of all the rods can be described as follows:

$$
\begin{aligned}
& g(x, y, z)=r_{x} \vee_{\alpha} r_{y} \vee_{\alpha} r_{z} \text { or } \\
& g(x, y, z)=\left(s_{y} \wedge_{\alpha} s_{z}\right) \vee_{\alpha}\left(s_{x} \wedge_{\alpha} s_{z}\right) \vee_{\alpha}\left(s_{x} \wedge_{\alpha} s_{y}\right)
\end{aligned}
$$

Note that all the parameters in the above lattice model can be made variable in space. Fig. 2 illustrates the construction of the regular lattice with some constant parameters (Fig. 2 a,b) and with the rod thickness controlled by the thresholds linearly changing along the horizontal axis (Fig. 2c). 

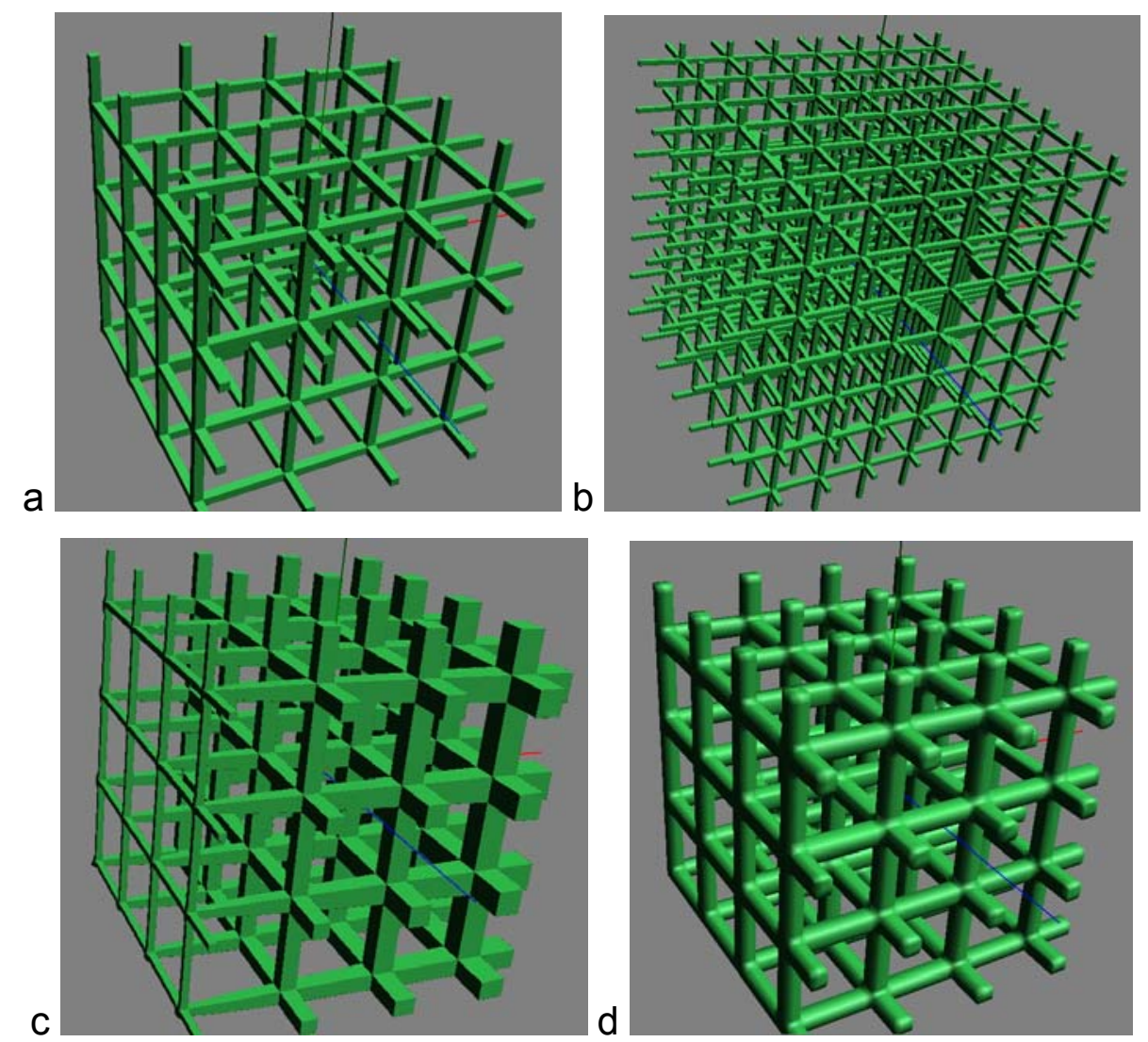

Figure 2. Function-based infinite regular lattice with (a) constant parameters; (b) double frequency (c) variable parameters - rod thickness grows linearly along one axis; (d) rods smoothed by blending.

The controlled blending versions of set-theoretic operations are applicable to FRep objects [PAS*95]. Instead of producing sharp edges, these operations result in smooth transitions between two given surfaces while a set operation is applied to two solids. The formulation of a blending operation is based on the displacement added to a standard R-function, for example for the blending intersection, we have:

$$
f_{1} \wedge_{b} f_{2}=\left(f_{1} \wedge_{\alpha} f_{2}\right)+\frac{a_{0}}{1+\left(\frac{f_{1}}{a_{1}}\right)^{2}+\left(\frac{f_{2}}{a_{2}}\right)^{2}}
$$

where $\wedge_{\alpha}$ stands for one of the R-functions defining the intersection (Eq.2-3) and the additional term defines the displacement with the parameters $a_{0}, a_{1}, a_{2}$ controlling the shape of the blend. For example, $a_{0}<0$ means the blend with removed material (chamfer). Fig. 2d illustrates an application of this operation to the rods construction, where the intersection operations of Eq. 5 are replaced by the blending intersection (Eq. 7). 

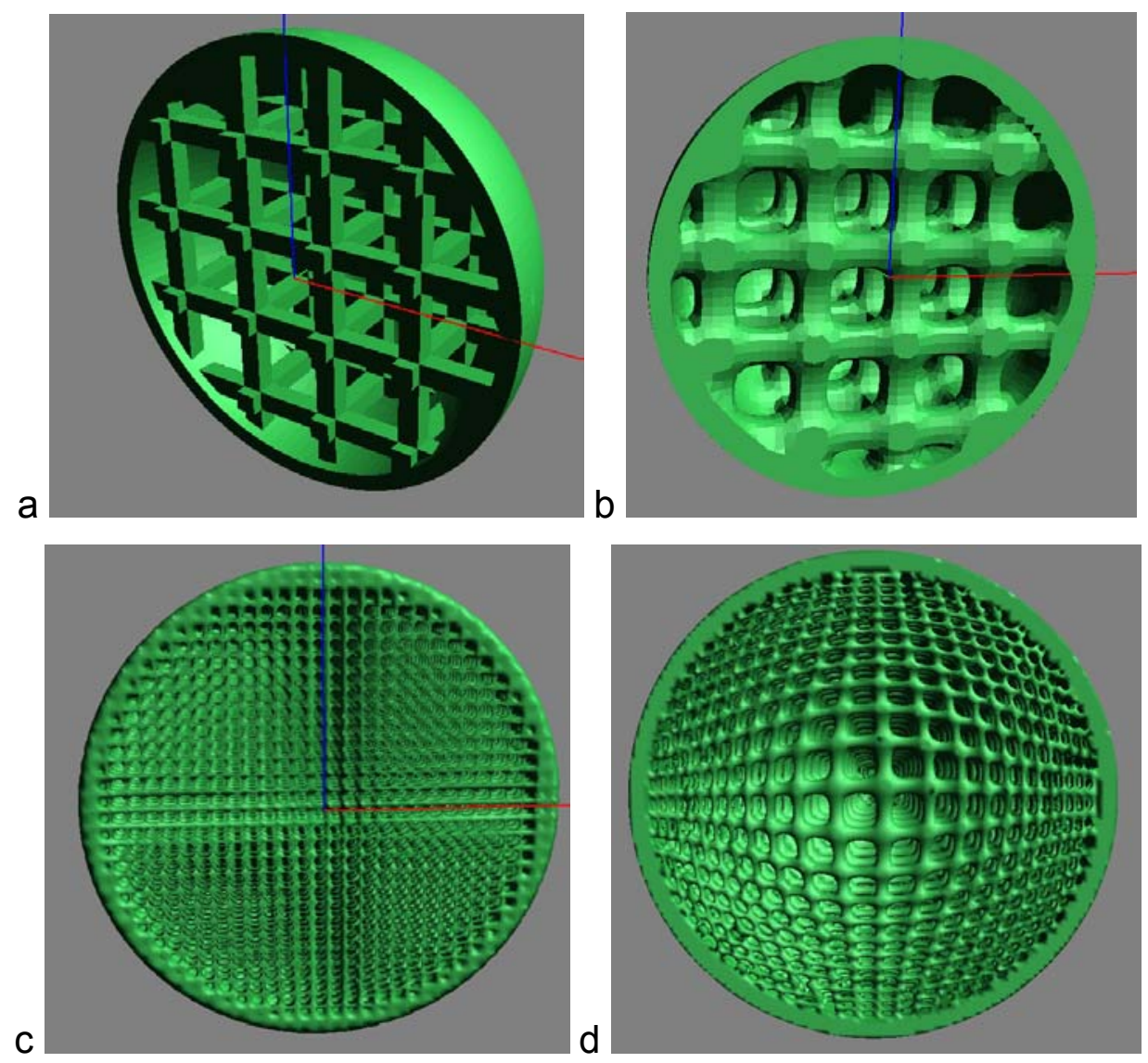

Figure 3. Combining microstructures with external shapes: (a) union of a spherical shell with a regular lattice; (b, c) blending union between the rods of the regular lattice, and between the rods and the spherical shell; (c) high-density lattice within a spherical shell; (d) lattice parameterization depending on the distance to the external surface.

It is important for the most of applications to be able to combine a microstructure with the given external shape of the object. Typically a thin shell of the object is created and the microstructure is truncated to fit inside the shell. These operations for the function $F$ defining the initial external shape can be described as follows:

$F_{o}(x, y, z)=F(x, y, z)-l_{o}$

defines the internal offset of the given shape, where $I_{0}>0$ is the offset threshold.

The shell of the object is defined as the subtraction of the offset from the initial shape:

$F_{s}(x, y, z)=F \backslash_{\alpha} F_{o}$,

the lattice within the object can be obtained by intersecting it with the given object:

$F_{g}=g \wedge_{\alpha} F$,

and finally the object shell with the microstructure inside has the defining function

$$
F_{m}(x, y, z)=F_{s} \vee_{\alpha} F_{g}=\left(F \backslash_{\alpha} F_{o}\right) \vee_{\alpha}\left(g \wedge_{\alpha} F\right)
$$




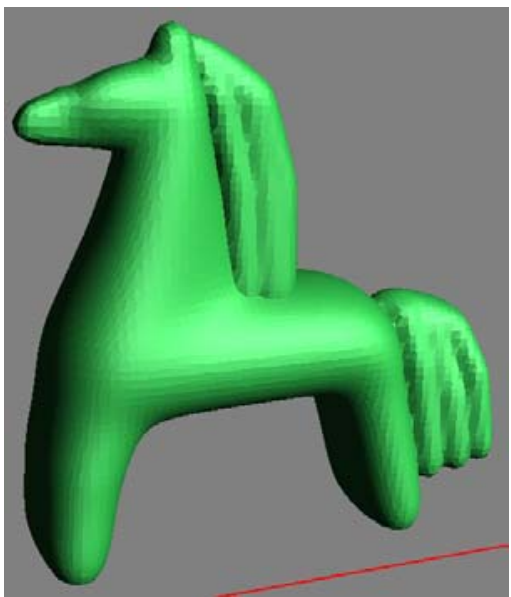

a

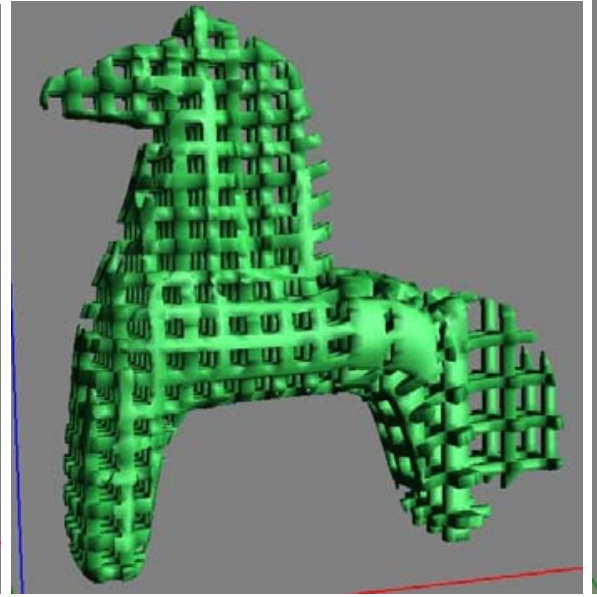

b

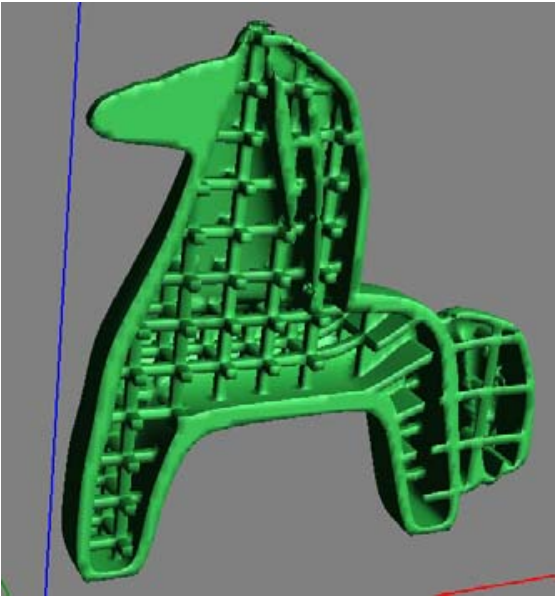

C

Figure 4. Operations on the given shape (a) and the microstructure: (b) intersection for the microstructure truncation; (c) union of the truncated microstructure with the object shell.

The above construction is illustrated by Fig. 3a, where the function $F$ defines a sphere. If the union operation in Eq. 8 is replaced by the blending union, the microstructure rods will be blended with the shell as shown in Figs. 3 b,c. A more complex shape (Fig. 4) can undergo the same operations. Note that the lattices in Figs. 4 b,c have non-uniform cell sizes (lattice frequency) depending on the coordinate $x$ value.

The lattice parameterization resulting in variable density and rod sizes can be made dependent not only on spatial coordinates, but on other factors. Thus, in biomedical engineering the lattice scaffolds has to be denser near the surface. This can be achieved by making lattice parameters dependable on the distance to the surface or, in the case of FRep object, on the defining function, which takes zero value on the boundary (see Fig. 3d).

\section{Modelling porous microstructures}

Porous media needs an approach different from the one suitable for modelling the regular rectangular lattices. As we could see from the survey of existing works, interconnected spherical pores with pseudo-random variations of sizes and positions are typically used in the porous structure analysis. We propose the following modelling procedure:

1) select a basic pore shape such as a sphere or an ellipsoid;

2) replicate the basic pore in space with variable parameters, if necessary;

3) subtract the replicated pores from the given initial shape;

4 ) introduce pseudo-random variations of the pores positions and sizes. 

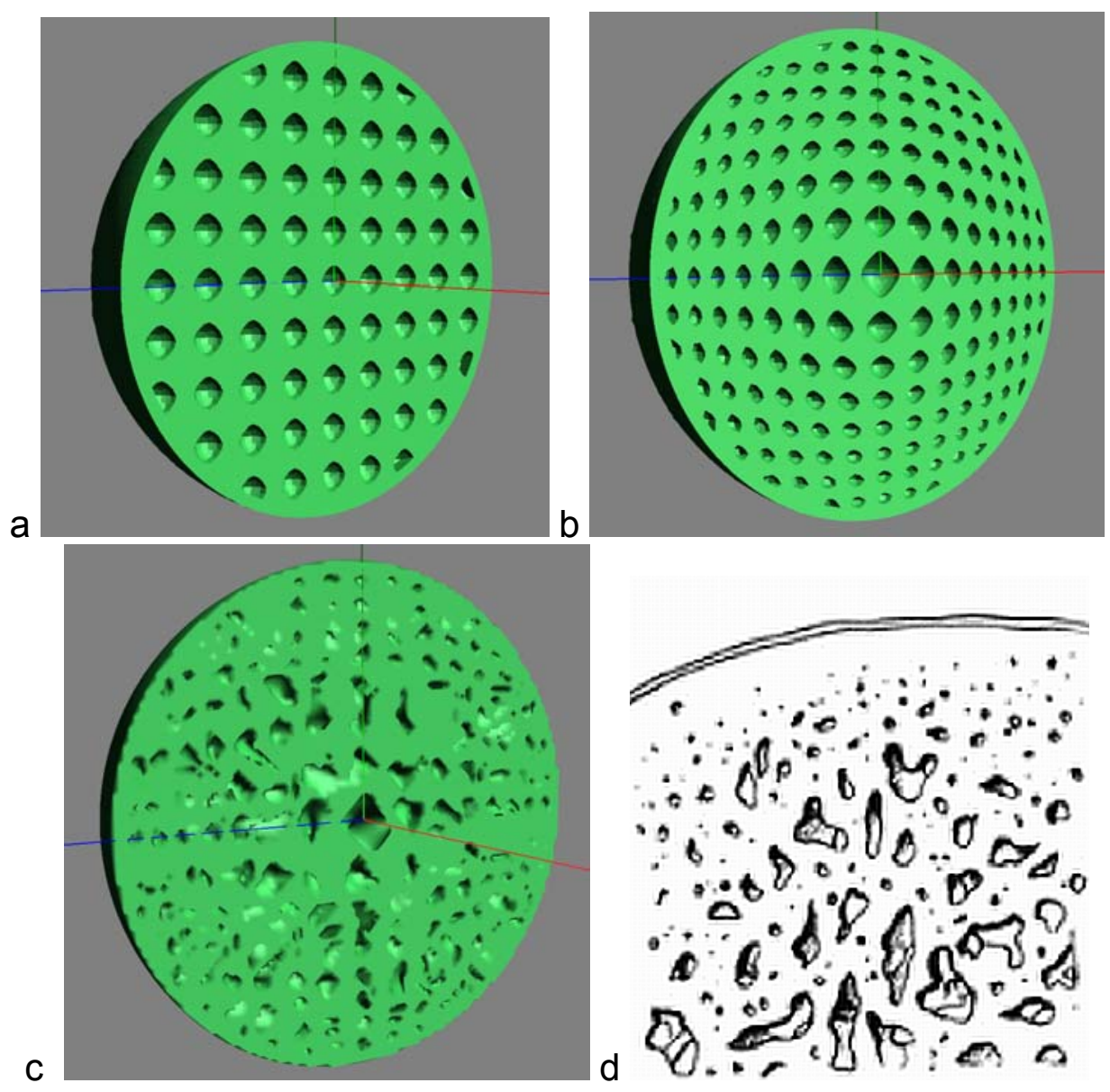

Figure 5. Function-based porous media modelling: (a) the basic pore replicated in space and subtracted from the initial shape; (b) the pore sizes made decreasing with the distance to the surface; (c) the pseudo-random variations of pores' parameters; (d) the cross section of a sample bone for comparison.

The proposed procedure is illustrated by Figs. 5 a-c. A basic pore with the defining function $F_{p}$ can be replicated in space (Fig. $5 a$ ) by the following space mapping:

$x^{\prime}=p_{x} \sin \left(q_{x} x\right)$

$y^{\prime}=p_{y} \sin \left(q_{y} x\right)$

$z^{\prime}=p_{z} \sin \left(q_{z} x\right)$

$F_{r}=F_{p}\left(x^{\prime}, y^{\prime}, z^{\prime}\right)$,

where $p_{x}>0$ specifies a linear scaling for the $x$-coordinate and $q_{x}>0$ controls the frequency of the basic pore replication along the $x$-axis. The replicated pores make actual cavities in the initial shape (sphere in Fig. 5) with the defining function $F$ through the set-theoretic subtraction:

$F_{m}(x, y, z)=F \backslash_{\alpha} F_{r}$

Similar to the case of lattices, a pore size can be made variable depending on the distance to the initial surface (Fig. 5b). Finally, the pseudo-random deformations of pores can be introduced by adding a solid noise function [G84, L89] to any of the model parameters. In the example of Fig. 5c, the Gardner's noise [G84] with different parameters was added by the algebraic summation of scale $p$, frequency $q$, and basic pore function $F_{p}$. The solid noise parameters were selected to provide the pore shapes similar to those in the sample 
bone cross-section (Fig. 5d). More sophisticated methods are definitely required to ensure the model adequacy to samples of natural porous media.

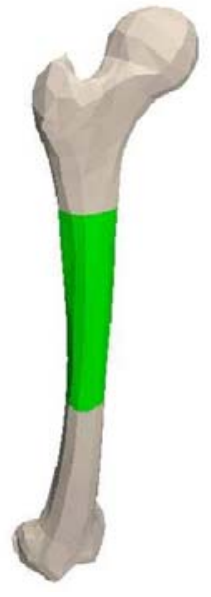

a

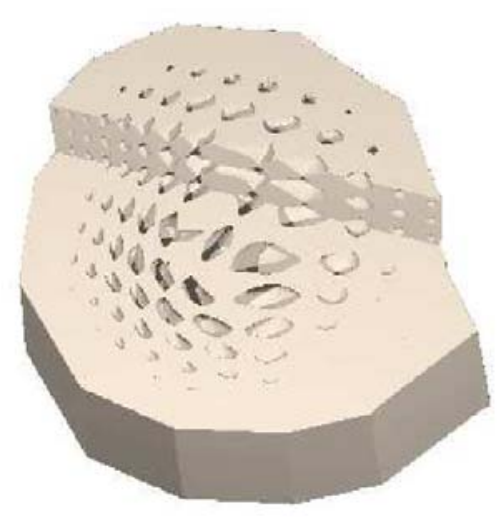

b

Figure 7. Modelling a bone structure: a) a polygonal bone model, green colour denotes the extracted segment of interest; (b) A cut-away section of the bone segment of interest showing the generated porous microstructure with the pore sizes decreasing with the distance to the bone surface.

The initial surface can be also provided in the form of a polygonal mesh or a voxel model composed of cross-sectional images. To provide the microstructure parameterization dependent on the defining function, one can involve the evaluation of the Euclidean distance function to the external surface. However, for such operations as blending of a lattice with a shell, the Euclidean distance has poor differential properties with $\mathrm{C}^{1}$ discontinuities at the medial axis points of the mesh. An alternative way of providing a real defining function for the mesh is its exact conversion to a differentiable scalar field based on the binary space partitioning [FPA08]. The use of such a conversion is illustrated in Fig. 7 by the model of the porous bone structure, where the scalar field obtained by the conversion procedure possesses the distance property and is used for scaling down pore sizes closer to the initial surface.

\section{Rendering and fabrication}

Application areas of microstructure modelling such as composite material design and biomedical tissue engineering require model rendering and manufacturing procedures. Typically a function-based model has to be converted to some auxiliary representations for subsequent rendering using modern graphics hardware and then for manufacturing on rapid prototyping or 3D printing equipment. The conversion to BRep involves an isosurface polygonization (tessellation) while the voxelization is needed to produce a voxel array representation. The disadvantages of both these auxiliary representations in the case of microstructure modelling were discussed in Section 1. A more promising approach can be called direct rendering and fabrication. 

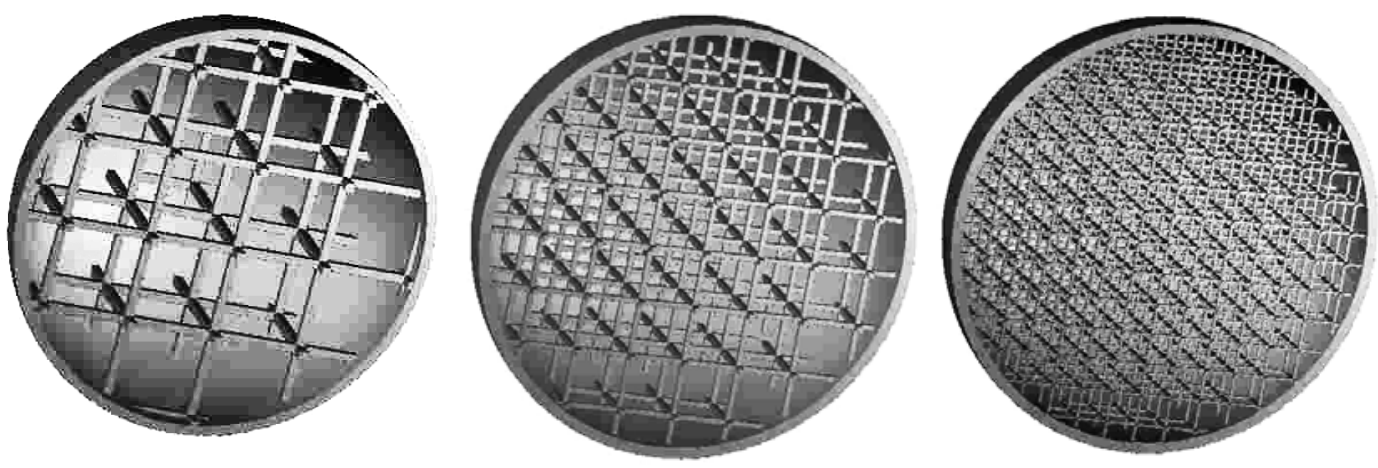

Figure 6. Ray-casting of a spherical thin shell with the internal regular lattice of different density; GPU rendering rate remains about 10 frames per second independent of the microstructure complexity (image size $\left.256^{2}\right)$.

Direct rendering of isosurfaces can be done with accelerated ray-tracing/ray-casting. In [FP08] it was shown that using of GPU allows direct rendering of relatively complex function-based models with interactive rates on modern graphics hardware. An example of ray-casting of the sphere with microstructures is shown in Fig. 6 . As the complexity of the function does not depend on the density of microstructures, the rendering time is almost the same for lattices with different densities on the given graphics hardware.
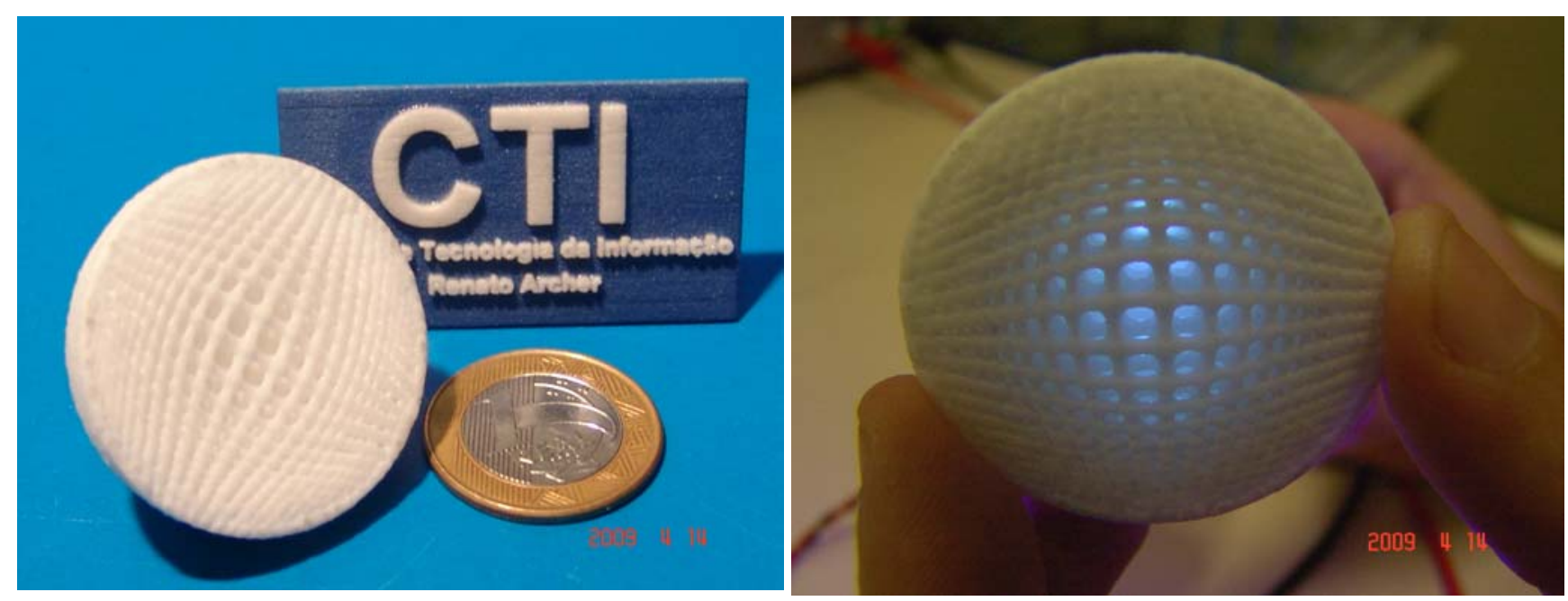

Figure 7. Model fabricated in polyamide material using a 3D Systems Sinterstation $\mathrm{HiQ}$ at the Centro de Tecnologia da Informação, Brasil.

Several of the FRep based microstructure models described above have been fabricated using a variety of digital fabrication machines and materials. For example, Fig. 7 shows a fabricated model of a lattice parametrized by the distance to the external surface (Fig. 3d). These models were printed at the Centro de Tecnologia da Informação in Brasil. The models were first polygonized and then output as STL files. The STL files were imported into the various software packages for driving the machine where they were checked for surface defects and then machine paths were generated based on layered slices of the STL and manufacturing constraints. Many of the models must also go through post processing to remove support structure and/or add additional strength. The STL file format created many issues for the slicing, path planning and fabrication of some of the fine microstructures. 
A much better approach to fabrication is to directly control the digital fabrication process using the FRep model at the resolution of any given machine without poor intermediate formats such as STL. This has been previously proposed [VML*08] and is an active ongoing focus of our research. However, obstacles presented by the proprietary nature of most digital fabrication technology, such as access to machine protocols and languages, require open hardware systems such as the Fab@Home or RepRap. Unfortunately, the resolution of these free and open source systems is currently not competitive with high end commercial machines necessary for fine resolution microstructures.

\section{Discussion and conclusions}

We have proposed two approaches based on different use of the periodic functions for modelling both lattice and porous microstructures within the FRep framework. In the case of lattices, these functions serve to directly define the point membership by analyzing the sign of the function. In the case of porous media, the periodic functions are used for space mapping (coordinates transformations) such that some basic shape can be infinitely replicated in space. A simple spherical pore was tested; however, any pore shape can be involved without any changes in the rest of the generation procedure.

The proposed models are extremely compact (all examples in this paper can be implemented in 10-20 lines of HyperFun [ACF* 99 ] or in 30-40 lines of $C$ language), while providing precise and spatially coherent models. The level of the model parameterization can be very high including parameter-dependent changes in object's topology. The FRep models of microstructures can be used as arguments for further set-theoretic, blending, offsetting, and other geometric operations. These models can be directly rendered using ray-tracing with interactive rates in the case of the GPU implementation.

The direct microstructure fabrication without generating any auxiliary BRep or voxel representations is one of the immediate subjects for our research and development. Another subject is the model adequacy analysis when we need to compare generated microstructures with those obtained by means of magnetic resonance imaging and other measurements of natural or artificial objects. As the model strength can be one of the design criteria, the strength analysis will be an area of our future research on the basis of the heterogeneous objects discretization and finite element meshes generation reported in FEA [KAP*03]. Finally, we intend to develop the function-based models for other types of microstructures such as octahedral lattices, natural branching and organic structures.

\section{Acknowledgements}

We would like to thank the support of Arnaldo Luis Lixandrão Filho, Paulo Inforçatti Neto, and Jorge Vicente Lopes da Silva at the Centro de Tecnologia da Informação - CTI in Brasil (they can be reached by email at arnaldo.filho@cti.gov.br), who helped make this work possible by digitally fabricating many models. 


\section{References}

[ACF*99] Adzhiev V., Cartwright R., Fausett E., Ossipov A., Pasko A., Savchenko V., HyperFun project: a framework for collaborative multidimensional F-rep modelling, Implicit Surfaces '99, Eurographics/ACM SIGGRAPH Workshop, J. Hughes and C. Schlick (Eds.), pp. 59-69, http://www.hyperfun.org

[B*97] Bloomenthal J. et al., Introduction to Implicit Surfaces, Morgan-Kaufmann, 1997, $332 \mathrm{p}$.

[C07] Chen Y., 3D Texture mapping for rapid manufacturing, Computer-Aided Design \& Applications, Vol. 4, No. 6, 2007, pp 761-771.

[CTS07] Chow H.N., Tan S.T., Sze W.S., Layered modelling of porous structures with Voronoi diagrams, Computer-Aided Design \& Applications. 2007, 4(1-4): 321-330.

[CYL05] Chua, C. K.; Yeong, W. Y.; Leong, K. F.: Rapid prototyping in tissue engineering: a state-of-the-art report, In: Virtual modelling and rapid manufacturing, edited by Bártolo, $\mathrm{P}$. J.: Taylor \& Francis, London, 2005.

[FP08] Fryazinov O., Pasko A., Interactive ray shading of FRep objects, WSCG'2008, Communications Papers proceedings, 2008, pp. 145-152.

[FPA08] Fryazinov O., Pasko A., Adzhiev V., An Exact Representation of Polygonal Objects by $C^{\wedge} 1$-continuous Scalar Fields Based on Binary Space Partitioning, Technical Report TRNCCA-2008-03, ISBN 1-85899-123-4, The National Centre for Computer Animation, Bournemouth University, UK, 2008, 12 p. (accepted to SMl'09 conference)

[G84] Gardner G., Simulation of natural scenes using textured quadric surfaces. Proceedings of SIGGRAPH '84, Computer Graphics 18, 3 (July 1984), 11-20.

[GSR05] V. Gervasi, A. Schneider, J. Rocholl, Geometry and procedure for benchmarking SFF and hybrid fabrication process resolution, Rapid Prototyping Journal, vol. 11, no. 1, 2005, pp. 4-8.

[H07] Hanna S., Inductive machine learning of optimal modular structures: estimating solutions using support vector machines, Artificial Intelligence for Engineering Design, Analysis and Manufacturing, vol. 21, No. 1, 2007, pp. 351-366.

[KAP*03] Kartasheva E., Adzhiev V., Pasko A., Fryazinov O., Gasilov V., Discretization of functionally based heterogeneous objects, 8th ACM Symposium on Solid Modelling and Applications, G. Elber and V. Shapiro (Eds.), ACM Press, 2003, pp. 145-156.

[KT08] X. Y. Kou, S. T. Tan, Heterogeneous object design: an integrated CAX perspective, in Heterogeneous Objects Modelling and Applications, Lecture Notes in Computer Science, vol. 4889, A. Pasko, V. Adzhiev, and P. Comninos (Eds.), Springer Verlag, 2008, pp. 42-59.

[L89] Lewis J. P., Algorithms for solid noise synthesis, SIGGRAPH'89, Proceedings of the 16th annual conference on Computer graphics and interactive techniques, 1989, pp. 263270.

[LS04] P. Lal, and W. Sun, Computer modelling approach for microsphere-packed bone scaffold, Computer-Aided Design, vol. 36, pp. 487-497, 2004.

[MH03] Mahdavi S., Hanna S., An evolutionary approach to microstructure optimisation of stereolithographic models, Proceedings of CEC2003, Congress on Evolutionary Computation, Institute of Electrical and Electronics Engineers, vol. 1, 2003, pp. 723-730. 
[NCL*05] Naing M. W., Chua C. K., Leong K. F., Wang Y., Fabrication of customised scaffolds using computer-aided design and rapid prototyping techniques, Rapid Prototyping Journal, vol. 11, No. 4, 2005, pp. 249-259.

[PAS*95] Pasko A., Adzhiev V., Sourin A., Savchenko V., Function representation in geometric modelling: concepts, implementation and applications, The Visual Computer, vol.11, No. 8, 1995, pp.429-446.

[R74] Rvachev V., Methods of Logic Algebra in Mathematical Physics, Naukova Dumka, Kiev, 1974 (in Russian).

[SRS*05] C. Schroeder, W. C. Regli, A. Shokoufandeh, and W. Sun, Computer-aided design of porous artifacts, Computer-Aided Design, vol. 37, pp. 339-353, 2005.

[S88] Shapiro V., Theory of R-functions and Applications: A Primer, Technical Report CPA88-3, Cornell University, 1988, 27 p.

[S07] Shapiro V., Semi-analytic geometry with R-functions, Acta Numerica 16, Cambridge University Press, 2007, pp. 239-303.

[SSN*05] W. Sun, B. Starly, J. Nam, A. Darling, Bio-CAD modelling and its applications in computer-aided tissue engineering, Computer-Aided Design, vol. 37, 2005, pp. 1097-1114.

[VAG*08] E. Verges, D. Ayala, S. Grau, D. Tost, 3D reconstruction and quantification of porous structures, Computers \& Graphics 32 (2008) 438- 444.

[VML ${ }^{*} 08$ ] Vilbrandt T., Malone E., Lipson H., Pasko A., Universal desktop fabrication, in Heterogeneous Objects Modelling and Applications, Lecture Notes in Computer Science, vol. 4889, A. Pasko, V. Adzhiev, and P. Comninos (Eds.), Springer Verlag, 2008, pp. 259284.

[WCR05] Wang, H., Chen, Y., Rosen, D. W., A hybrid geometric modelling method for large scale conformal cellular structures, ASME International Design Engineering Technical Conferences and Computers and Information in Engineering Conferences, 2005, DETC2005-85366. 